\title{
Ternary Menger Algebras: A Generalization of Ternary Semigroups
}

\author{
Anak Nongmanee ${ }^{1}$ iD and Sorasak Leeratanavalee ${ }^{2, *(D)}$ \\ 1 M.S. Program in Mathematics, Department of Mathematics, Faculty of Science, Chiang Mai University, \\ Chiang Mai 50200, Thailand; anak_nongmanee@cmu.ac.th \\ 2 Research Center in Mathematics and Applied Mathematics, Department of Mathematics, Faculty of Science, \\ Chiang Mai University, Chiang Mai 50200, Thailand \\ * Correspondence: sorasak.1@cmu.ac.th
}

check for

updates

Citation: Nongmanee, A.;

Leeratanavalee, S. Ternary Menger Algebras: A Generalization of Ternary Semigroups. Mathematics 2021, 9, 553. https://doi.org/doi:10.3390/ math9050553

Academic Editor: Michele Elia

Received: 19 January 2021

Accepted: 28 February 2021

Published: 5 March 2021

Publisher's Note: MDPI stays neutral with regard to jurisdictional claims in published maps and institutional affiliations.

Copyright: (c) 2021 by the authors. Licensee MDPI, Basel, Switzerland. This article is an open access article distributed under the terms and conditions of the Creative Commons Attribution (CC BY) license (https:/ / creativecommons.org/licenses/by/ $4.0 /)$.

\begin{abstract}
Let $n$ be a fixed natural number. Menger algebras of rank $n$, which was introduced by Menger, K., can be regarded as the suitable generalization of arbitrary semigroups. Based on this knowledge, an interesting question arises: what a generalization of ternary semigroups is. In this article, we first introduce the notion of ternary Menger algebras of rank $n$, which is a canonical generalization of arbitrary ternary semigroups, and discuss their related properties. In the second part, we establish the so-called a diagonal ternary semigroup which its operation is induced by the operation on ternary Menger algebras of rank $n$ and then investigate their interesting properties. Moreover, we introduce the concept of homomorphism and congruences on ternary Menger algebras of rank $n$. These lead us to study the quotient ternary Menger algebras of rank $n$ and to investigate the homomorphism theorem for ternary Menger algebra of rank $n$ with respect to congruences. Furthermore, the characterization of reduction of ternary Menger algebra into Menger algebra is presented.
\end{abstract}

Keywords: ternary Menger algebra; diagonal ternary semigroup; congruence; isomorphism theorem; reduction

\section{Introduction}

In 1946, Menger, K. [1] studied the algebraic properties of the composition of multiplace functions. In addition, the property of the composition, which is called superassociative law, was studied in both primary and advanced ways. By using this idea, the concept of Menger algebras of rank $n$, for all natural numbers $n$ (sometimes, it is called superassociative algebras) was presented. From now on, we assume that $n$ be a natural number. A Menger algebra of rank $n$ (see [2]) $(T, o)$ is a pair of a nonempty set $T$ of elements and an $(n+1)$-ary operation $o$ on $T$ which satisfies the superassociative law, i.e.,

$$
o\left(o\left(x, y_{1}, \ldots, y_{n}\right), z_{1}, \ldots, z_{n}\right)=o\left(x, o\left(y_{1}, z_{1}, \ldots, z_{n}\right), \ldots, o\left(y_{n}, z_{1}, \ldots, z_{n}\right)\right)
$$

for all $x, y_{i}, z_{i} \in T, i=1, \ldots, n$. If we set $n=1$, then the identity (1) is reduced to the associative law and a Menger algebra of rank 1 forms an arbitrary semigroup.

The theory of Menger algebras of rank $n$ and its applications are developed by Dudek, W. A. and Trokhimenko, V. S. who studied the so-called principal v-congruences on Menger algebras of rank $n$, which are the generalizations of the principal right and left congruences on arbitrary semigroups (see [3]). Up to 2017, they presented the abstract characterization of various types of Menger algebras of $n$-place functions allowing certain permutations of variables (see [4]), while Trokhimenko, V. S. [5] introduced the so-called v-regular Menger algebras and investigated its related properties. Moreover, regular elements and Green's relations in Menger algebras of terms were studied by Denecke, K. in 2006 [6]. According to transformations semigroups, Clifford, A. H. [7] introduced the important class of transformations the so-called translations in 1950. Up to 1964, Schein, B. M. [8] studied 
the problem of characterizing inner translations on semigroups. Based on these knowledge, Kumduang, T. and Leeratanavalee, S. introduced the notion of the left translations for Menger algebras of rank $n$ and studied some of its algebraic properties. In addition, they also studied the isomomorphism theorem for Menger algebras of rank $n$ (see [9]). In 2020 [10], they showed that every abstract Menger hyperalgebra, which is a canonical generalization of semihypergroups, can be represented by multivalued full $n$-ary functions. For more information related to Menger algebras see [11].

In 1932, Lehmer, D. H. [12] introduced the definition and theory of ternary semigroups. Previously, in 1904, Kanser, E. [13] presented the so-called $n$-ary algebras, which is the extension of the group concept (cf. [14]). According to ternary algebraic structures and its generalization, the so-called $n$-ary algebraic structures, raise certain hopes in view of their applications in Physics. The quark model inspired a particular brand of ternary algebraic structures, intended to describe the non-observability of the isolated quarks as the phenomenon of the algebraic confinement (cf. [15]). Moreover, one of the first such endeavors has been presented by Numbu, Y. [16] in 1973 and known as the so-called Nambu mechanics. For further physical applications, see $[17,18]$.

The important area of research of ternary structures (cf. [19]) are ternary algebras, ternary linear algebras, normed ternary linear algebras, involutive ternary linear algebras, topological ternary linear algebras, $C^{*}$-ternary algebras, and Banach ternary algebras (see [20-26]). Furthermore, the ternary algebraic structures and the $n$-ary algebraic structures are considered in the theory of functional equations (see [27-30]). In particular, consideration such structures in the stability of functional equations (see [31-33]).

The notion of ternary semigroups was known for the first time by Banach, S. (cf. [34,35]) who showed (by an example) that a ternary semigroup does not necessarily reduce to an ordinary semigroup. A ternary semigroup $(T, \diamond)$ is a groupoid together with a ternary operation $\diamond: T^{3} \longrightarrow T$, which is satisfies the ternary associative law, i.e.,

$$
\diamond(\diamond(a, b, c), d, e)=\diamond(a, \diamond(b, c, e), e)=\diamond(a, b, \diamond(c, d, e)) \quad \text { for all } a, b, c, d, e \in T .
$$

On the other hand, a ternary semigroup is considered to be a special case of an $n$-ary semigroup, which is a natural generalization of the notion of ternary semigroups, for $n=3$ (cf. [36]).

Analogous to the theory of semigroup, the algebraic structure of ternary semigroups and its related properties are studied by many mathematicians in various topics. In 1955, Los, J. [35] proved that every ternary semigroup can be embedded in a semigroup and studied some properties of ternary semigroups. Up to 1965, Sioson F. M. [37] studied an ideal theory in ternary semigroups. Moreover, he also defined regular ternary semigroups and characterized it by the notion of quasi-ideals. Sheeja, G. and Sri Bala, S. [38] investigated the so-called orthodox ternary semigroups. Vagner, V. [39] studied semiheaps which are a ternary system under an operation satisfying a different type of ternary associative law in 1953. Next, in 1983, the theory of ternary semigroups and semiheaps were developed by Santiago, M. L. in [40]. In 1940, Post, E. L. [41] constructed the so-called covering groups (cf. [42]). Based on the concept of covering groups, Santiago, M. L. and Sri Bala, S. [43] constructed the semigroup $S_{T}$ such that a ternary semigroup $T$ embedded in $S_{T}$, and investigated some related properties in 2010. Recently, congruences and some different types of congruences on ternary semigroups are presented in [14,34,44,45].

To the main results of the article, let us consider on Menger algebras $(T, o)$ of rank $n$. If we set $n=2$ for the identity (1), then we get $o: T^{3} \longrightarrow T$ which is ternary operation such that satisfying the identity

$$
o\left(o\left(x, y_{1} y_{2}\right), z_{1}, z_{2}\right)=o\left(x, o\left(y_{1}, z_{1}, z_{2}\right), o\left(y_{2}, z_{1}, z_{2}\right)\right)
$$

for all $x, y_{1}, y_{2}, z_{1}, z_{2} \in T$. We see that the identities (2) and (3) are not the same thing, which means that the ternary operation $o$ on $T$ does not satisfy the ternary associative law. Consequently, Menger algebra of rank $n$ is not a generalization of arbitrary ternary 
semigroups. Therefore, it will be very interesting to see what a generalization of arbitrary ternary semigroups is.

In this paper, we first introduce the notion of ternary Menger algebras of rank $n$ which can be regarded as a canonical generalization of arbitrary ternary semigroups in a different sense from $n$-ary semigroups. In Section 2 , we establish a new ternary algebraic structure the so-called a diagonal ternary semigroup. Moreover, its interesting algebraic properties are investigated. In Section 3, we define a homomorphism and congruences on ternary Menger algebras of rank $n$. Furthermore, we also extend some well-known results related to congruences from arbitrary ternary semigroups to its extension. In Section 4, we complete the paper with the characterization of reduction of ternary Menger algebra of rank $n$ into Menger algebra of rank $n$.

\section{Ternary Menger Algebra and Diagonal Ternary Semigroup}

In this section, we introduce the notion of a ternary Menger algebra of rank $n$, which is a canonical generalization of arbitrary ternary semigroups, and give some of its examples. Moreover, we define a new ternary operation which is induced by the operation on ternary Menger algebra of rank $n$ and hence we obtain the so-called a diagonal ternary semigroup.

Definition 1. $A(2 n+1)$-ary groupoid $(T, \bullet)$, i.e., a nonempty set $T$ with one $(2 n+1)$-ary operation $\bullet: T^{2 n+1} \longrightarrow T$, is called a ternary Menger algebra of rank $n$ (a ternary superassociative algebra) if it satisfies the so-called ternary superassociative law:

$$
\begin{aligned}
\bullet\left(\bullet\left(a, b_{1}, . ., b_{n}, c_{1}, \ldots, c_{n}\right), d_{1}, \ldots, d_{n}, e_{1}, \ldots, e_{n}\right) \\
=\bullet\left(a, \bullet\left(b_{1}, c_{1}, \ldots, c_{n}, d_{1}, \ldots, d_{n}\right), \ldots, \bullet\left(b_{n}, c_{1}, \ldots, c_{n}, d_{1}, \ldots, d_{n}\right), e_{1}, \ldots, e_{n}\right) \\
=\bullet\left(a, b_{1}, \ldots, b_{n}, \bullet\left(c_{1}, d_{1}, \ldots, d_{n}, e_{1}, \ldots, e_{n}\right), \ldots, \bullet\left(c_{n}, d_{1}, \ldots, d_{n}, e_{1}, \ldots, e_{n}\right)\right) .
\end{aligned}
$$

Please note that if $n=1$, then we obtain a ternary Menger algebra of rank 1 (a ternary semigroup). For convenience, we write $a\left[b_{1} \ldots b_{n} c_{1} \ldots c_{n}\right]$ or $a[\bar{b} \bar{c}]$ instead of $\bullet\left(a, b_{1}, . ., b_{n}, c_{1}, \ldots, c_{n}\right)$. In case $b_{1}=\ldots=b_{n}$ and $c_{1}=\ldots=c_{n}$, we write $a\left[b^{n} c^{n}\right]$ instead of $\bullet\left(a, b_{1}, . ., b_{n}, c_{1}, \ldots, c_{n}\right)$ and it will be interpreted as the result of the operation $\bullet$ applied to the elements $a_{1}, b_{1}, \ldots, b_{n}, c_{1}, \ldots$, $c_{n} \in T$. By this convention, the above ternary superassociative law can be written as the following:

$$
\begin{aligned}
a\left[b_{1} \ldots b_{n} c_{1} \ldots c_{n}\right]\left[d_{1} \ldots d_{n} e_{1} \ldots e_{n}\right] & =a\left[b_{1}\left[c_{1} \ldots c_{n} d_{1} \ldots d_{n}\right] \ldots b_{n}\left[c_{1} \ldots c_{n} d_{1} \ldots d_{n}\right] e_{1} \ldots e_{n}\right] \\
& =a\left[b_{1} \ldots b_{n} c_{1}\left[d_{1} \ldots d_{n} e_{1} \ldots e_{n}\right] \ldots c_{n}\left[d_{1} \ldots d_{n} e_{1} \ldots e_{n}\right]\right],
\end{aligned}
$$

or its shortly form as

$$
a[\bar{b} \bar{c}][\bar{d} \bar{e}]=a\left[b_{1}[\bar{c} \bar{d}] \ldots b_{n}[\bar{c} \bar{d}] \bar{e}\right]=a\left[\bar{b} c_{1}[\bar{d} \bar{e}] \ldots c_{n}[\bar{d} \bar{e}]\right] .
$$

Example 1. (i) Let $T=\mathbb{Z} \times \mathbb{Z}=\{(x, y): x, y \in \mathbb{Z}\}$. Define $a(2 n+1)$-ary operation $\bullet$ on $T$ by

$$
(x, y)\left[\left(x_{1}, y_{1}\right) \ldots\left(x_{n}, y_{n}\right)\left(a_{1}, b_{1}\right) \ldots\left(a_{n}, b_{n}\right)\right]=\left(x, b_{n}\right) \text { for all } a_{i}, b_{i}, x, x_{i}, y, y_{i} \in \mathbb{Z}, i=1, \ldots, n \text {. }
$$

Then $(T, \bullet)$ is a ternary Menger algebra of rank $n$.

(ii) Consider a set $\mathbb{N}_{+}$of all non-zero natural numbers together with $a(2 n+1)$-ary operation - defined by

$$
x[\bar{y} \bar{z}]=x \cdot \operatorname{gcd}\{\bar{y}\} \cdot \operatorname{gcd}\{\bar{z}\} \quad \text { for all } x, y_{i}, z_{i} \in \mathbb{N}_{+}, i=1, \ldots, n,
$$

where and $\operatorname{gcd}\{\}$ mean a usual (binary) multiplication and the greatest common divisor, respectively. Then $\left(\mathbb{N}_{+}, \bullet\right)$ forms a ternary Menger algebra of rank $n$.

(iii) The set $\mathbb{R}^{+}$of all positive real numbers under $a(2 n+1)$-ary operation defined by

$$
x[\bar{y} \bar{z}]=x \cdot \sqrt[n]{y_{1} \cdots y_{n} \cdot z_{1} \cdots z_{n}} \quad \text { for all } x, y_{i}, z_{i} \in \mathbb{R}^{+}, i=1, \ldots, n,
$$

where $\cdot$ is a usual (binary) multiplication. Then $\left(\mathbb{R}^{+}, \bullet\right)$ is a ternary Menger algebra of rank $n$.

(iv) Let $\mathbb{R}$ be the set of all real numbers with a $(2 n+1)$-ary operation defined by 


$$
x[\bar{y} \bar{z}]=x+\frac{y_{1}+\ldots+y_{n}+z_{1}+\ldots+z_{n}}{n} \quad \text { for all } x, y_{i}, z_{i} \in \mathbb{R}, i=1, \ldots, n
$$

where + is a usual (binary) addition. Then $(\mathbb{R}, \bullet)$ forms a ternary Menger algebra of rank $n$.

(v) Consider the set $O^{n}(X)$ of all $n$-ary operations which are defined on the set $X$. Define a $(2 n+1)$-ary operation $S^{2 n, X}: O^{n}(X)^{2 n+1} \longrightarrow O^{n}(X)$ by, for each $f, \bar{g}, \bar{h} \in O^{n}(X)$,

$$
S^{2 n, X}(f, \bar{g}, \bar{h})(\bar{x})=f(\bar{g}(\bar{h}(\bar{x}))) \quad \text { for all } x_{i} \in X, i=1, \ldots, n .
$$

Therefore $\left(O^{n}(X), S^{2 n, X}\right)$ forms a $(2 n+1)$-ary groupoid. Moreover, it is easy to check that $\left(O^{n}(X), S^{2 n, X}\right)$ satisfies the following identities:

$$
\begin{aligned}
& \text { (C1) } \begin{aligned}
& \tilde{S}^{2 n}\left(\tilde{S}^{2 n}(\alpha, \bar{\beta}, \bar{\gamma}), \bar{\lambda}, \bar{\mu}\right) \approx \tilde{S}^{2 n}\left(\alpha, \tilde{S}^{2 n}\left(\beta_{1}, \bar{\gamma}, \bar{\lambda}\right), \ldots, \tilde{S}^{2 n}\left(\beta_{n}, \bar{\gamma}, \bar{\lambda}\right), \bar{\mu}\right) \\
& \approx \tilde{S}^{2 n}\left(\alpha, \bar{\beta}, \tilde{S}^{2 n}\left(\gamma_{1}, \bar{\lambda}, \bar{\mu}\right), \ldots, \tilde{S}^{2 n}\left(\gamma_{n}, \bar{\lambda}, \bar{\mu}\right)\right) \\
\text { (C2) } & \tilde{S}^{2 n}\left(\alpha, \delta_{1}, \ldots, \delta_{n}, \delta_{n+1}, \ldots, \delta_{2 n}\right)=\alpha
\end{aligned} \\
& \text { (C3) } \tilde{S}^{2 n}\left(\delta_{i}, \bar{\beta}, \bar{\gamma}\right)= \begin{cases}\beta_{i} & \text { if } i=1,2, \ldots, n \\
\gamma_{i-n} & \text { if } i=n+1, n+2, \ldots, 2 n,\end{cases}
\end{aligned}
$$

where $\tilde{S}^{2 n}$ is an $(2 n+1)$-ary operation symbol corresponding to the $(2 n+1)$-ary operations $S^{n}$, $\delta_{i}, i=1,2, \ldots ., 2 n$ are nullary operation symbols and $\alpha, \bar{\beta}, \bar{\gamma}, \bar{\lambda}, \bar{\mu}$ are variables.

Indeed, for each $f, \bar{g}, \bar{h}, \bar{p}, \bar{q} \in O^{n}(X)$ we have

$$
\begin{aligned}
S^{2 n, X}\left(S^{2 n, X}(f, \bar{g}, \bar{h}), \bar{p}, \bar{q}\right)(\bar{x}) & =S^{2 n, X}(f, \bar{g}, \bar{h})(\bar{p}(\bar{q}(\bar{x}))) \\
& =f(\bar{g}(\bar{h}(\bar{p}(\bar{q}(\bar{x}))))) \\
& =f\left(g_{1}(\bar{h}(\bar{p}(\bar{q}(\bar{x})))), \ldots, g_{n}(\bar{h}(\bar{p}(\bar{q}(\bar{x}))))\right) \\
& =S^{2 n, X}\left(f, S^{2 n, X}\left(g_{1}, \bar{h}, \bar{p}\right), \ldots, S^{2 n, X}\left(g_{n}, \bar{h}, \bar{p}\right), \bar{q}\right)(\bar{x})
\end{aligned}
$$

Similarly, $S^{2 n, X}\left(S^{2 n, X}(f, \bar{g}, \bar{h}), \bar{p}, \bar{q}\right)(\bar{x})=S^{2 n, X}\left(f, \bar{g}, S^{2 n, X}\left(h_{1}, \bar{p}, \bar{q}\right), \ldots, S^{2 n, X}\left(h_{n}, \bar{p}, \bar{q}\right)\right)(\bar{x})$. Hence $S^{2 n, X}$ satisfies (C1), which means that $S^{2 n, X}$ is ternary superassociative. Consequently, $\left(O^{n}(X), S^{2 n, X}\right)$ forms a ternary Menger algebra of rank $n$. Furthermore, it is easily seen that $S^{2 n, X}$ satisfies (C2) and (C3).

Remark 1. Let $T$ be a Menger algebra of rank $n$ with an $(n+1)$-ary operation $\left(x, y_{1}, \ldots, y_{n}\right) \mapsto$ $o\left(x, y_{1}, \ldots, y_{n}\right)$. Then $T$ under the $(2 n+1)$-ary operation defined by $\left(x, y_{1}, \ldots, y_{n}, z_{1}, \ldots, z_{n}\right) \mapsto$ $o\left(o\left(x, y_{1}, \ldots, y_{n}\right), z_{1}, \ldots, z_{n}\right)$ is a ternary Menger algebra of rank $n$, while a ternary Menger algebra of rank $n$ does not necessarily reduce to a Menger algebra of rank $n$.

Example 2. Consider a set $T=\{-i, i\}$ under the ternary multiplication of complex numbers is a ternary Menger algebra of rank 1 while $T$ with usual (binary) multiplication is not a Menger algebra of rank 1 .

Now, we define a new ternary operation induced by a $(2 n+1)$-ary operation of a ternary Menger algebra of rank $n$, then a nonempty base set of a ternary Menger algebra under this operation forms a ternary semigroup.

Proposition 1. Let $(T, \bullet)$ be a ternary Menger algebra of rank $n$. Define a ternary operation $*: T^{3} \longrightarrow T$ by

$$
*(a, b, c)=a\left[b^{n} c^{n}\right] \text { for all } a, b, c \in T,
$$

then $(T, *)$ forms a ternary semigroup. 
Proof. Indeed, for each $a, b, c, d, e \in T$ we have

$$
\begin{aligned}
*(*(a, b, c), d, e) & =*\left(a\left[b^{n} c^{n}\right], d, e\right) \\
& =a\left[b^{n} c^{n}\right]\left[d^{n} e^{n}\right] \\
& =a\left[b\left[c^{n} d^{n}\right] \ldots b\left[c^{n} d^{n}\right] e^{n}\right] \\
& =*(a, *(b, c, d), e) \quad \text { and } \\
*(*(a, b, c), d, e) & =*\left(a\left[b^{n} c^{n}\right], d, e\right) \\
& =a\left[b^{n} c^{n}\right]\left[d^{n} e^{n}\right] \\
& =a\left[b^{n} c\left[d^{n} e^{n}\right] \ldots c\left[d^{n} e^{n}\right]\right] \\
& =*(a, b, *(c, d, e)),
\end{aligned}
$$

which imply that $*$ is ternary associative. Therefore $(T, *)$ is a ternary semigroup.

We call the ternary semigroup $T$ under this operation, a diagonal ternary semigroup. Now, we introduce some special elements on a ternary Menger algebra, and show the relationship between a diagonal ternary semigroup and a ternary Menger algebra of rank $n$.

Definition 2. An element e of a ternary Menger algebra $T$ of rank $n$ is said to be a

(i) left diagonal unit if $e\left[e^{n} x^{n}\right]=x$ for all $x \in T$;

(ii) right diagonal unit if $x\left[e^{n} e^{n}\right]=x$ for all $x \in T$;

(iii) lateral diagonal unit if $e\left[x^{n} e^{n}\right]=x$ for all $x \in T$;

(iv) two-side diagonal unit if $e\left[e^{n} x^{n}\right]=x=x\left[e^{n} e^{n}\right]$ for all $x \in T$;

(v) diagonal unit if $e\left[e^{n} x^{n}\right]=x\left[e^{n} e^{n}\right]=e\left[x^{n} e^{n}\right]=x$ for all $x \in T$.

Definition 3. Let $(T, \bullet)$ be a ternary Menger algebra of rank $n$ and $a \in T$. Then $a$ is called idempotent, if $a\left[a^{n} a^{n}\right]=a$.

A ternary Menger algebra is called band, if every element of $T$ is idempotent.

Example 3. Let $T$ be a nonempty set and let $a \in T$ be fixed. If we define $a(2 n+1)$-ary operation - by

$$
x[\bar{y} \bar{z}]=a \quad \text { for all } x, y_{i}, z_{i} \in T, i=1, \ldots, n,
$$

then $(T, \bullet)$ forms a ternary Menger algebra of rank $n$ with an idempotent element $a$.

Example 4. $A(2 n+1)$-ary groupoid $(T, \bullet)$ together with the operation

$$
x[\bar{y} \bar{z}]=x \quad \text { for all } x, y_{i}, z_{i} \in T, i=1, \ldots, n
$$

is a ternary Menger algebra of rank $n$. Moreover, all elements in $T$ are idempotent and right diagonal unit, while $T$ has no left diagonal unit.

Example 5. Consider the set $\mathbb{N}_{+}$of all non-zero natural numbers under a $(2 n+1)$-ary operation - defined by

$$
x[\bar{y} \bar{z}]=\min \{x, \bar{y}, \bar{z}\} \quad \text { for all } x, y_{i}, z_{i} \in \mathbb{N}_{+}, i=1, \ldots, n
$$

forms a ternary Menger algebra of rank $n$ with a diagonal unit 1. Furthermore, the diagonal ternary semigroup of $\left(\mathbb{N}_{+}, \bullet\right)$ is commutative, i.e.,

$$
*\left(x_{1}, x_{2}, x_{3}\right)=*\left(x_{\sigma(1)}, x_{\sigma(2)}, x_{\sigma(3)}\right)
$$

for all permutation $\sigma$ of $\{1,2,3\}$ and $x_{1}, x_{2}, x_{3} \in \mathbb{N}_{+}$.

Proposition 2. Let $(T, \bullet)$ be a ternary Menger algebra of rank $n$ which has a two-side diagonal unit $e$. Then every element $x \in T$ satisfying $e=e\left[x^{n} x^{n}\right]$ is also a two-side diagonal unit. 
Proof. Indeed, for each $y \in T$ we obtain

$$
\begin{aligned}
& y=y\left[e^{n} e^{n}\right]=y\left[e^{n} e\left[x^{n} x^{n}\right] \ldots e\left[x^{n} x^{n}\right]\right]=y\left[e^{n} e^{n}\right]\left[x^{n} x^{n}\right]=y\left[x^{n} x^{n}\right] \text { and } \\
& y=e\left[e^{n} y^{n}\right]=e\left[e\left[x^{n} x^{n}\right] \ldots e\left[x^{n} x^{n}\right] y^{n}\right]=e\left[e^{n} x^{n}\right]\left[x^{n} y^{n}\right]=x\left[x^{n} y^{n}\right] .
\end{aligned}
$$

Hence $x$ is a two-side diagonal unit.

Proposition 3. Let $(T, \bullet)$ be a ternary Menger algebra of rank $n$ which has a left diagonal unit $e$ and for each $x, y \in T$ there exists an element $z \in T$ satisfying $e=z\left[x^{n} y^{n}\right]$. Then the diagonal ternary semigroup $(T, *)$ of $T$ is left cancellative., i.e.,

$$
*(x, y, a)=*(x, y, b) \Longrightarrow a=b \text { for all } a, b, x, y \in T .
$$

Proof. Assume that $*(x, y, a)=*(x, y, b)$ for all $a, b, x, y \in T$. By our assumption, we have $x\left[y^{n} a^{n}\right]=x\left[y^{n} b^{n}\right]$. It implies that

$$
\begin{aligned}
a & =e\left[e^{n} a^{n}\right] \\
& =e\left[z\left[x^{n} y^{n}\right] \ldots z\left[x^{n} y^{n}\right] a^{n}\right] \\
& =e\left[z^{n} x\left[y^{n} a^{n}\right] \ldots x\left[y^{n} a^{n}\right]\right] \\
& =e\left[z^{n} x\left[y^{n} b^{n}\right] \ldots x\left[y^{n} b^{n}\right]\right] \\
& =e\left[z\left[x^{n} y^{n}\right] \ldots z\left[x^{n} y^{n}\right] b^{n}\right] \\
& =e\left[e^{n} b^{n}\right] \\
& =b .
\end{aligned}
$$

Hence the diagonal ternary semigroup $(T, *)$ is left cancellative.

Proposition 4. For a diagonal ternary semigroup $(T, *)$ of a ternary Menger algebra $(T, \bullet)$ of rank $n$,

$$
*(x, y, z)[\bar{a} \bar{b}]=*(x, y, z[\bar{a} \bar{b}]) \quad \text { for all } a_{i}, b_{i}, x, y, z \in T, i=1, \ldots, n \text {. }
$$

Proof. Indeed, for $a_{i}, b_{i}, x, y, z \in T, i=1, \ldots, n$ we have

$$
\begin{aligned}
*(x, y, z)[\bar{a} \bar{b}] & =x\left[y^{n} z^{n}\right][\bar{a} \bar{b}] \\
& =x\left[y^{n} z[\bar{a} \bar{b}] \ldots z[\bar{a} \bar{b}]\right] \\
& =*(x, y, z[\bar{a} \bar{b}]) .
\end{aligned}
$$

This completes the proof.

Previously, we have shown the relationship between a ternary Menger algebra and a diagonal ternary semigroup, i.e., if we have a ternary Menger algebra of rank $n$, then we obtain the so-called a diagonal ternary semigroup. On the other hand, if we have a ternary semigroup satisfying the conditions given below, then it will be a diagonal ternary semigroup of some ternary Menger algebras of rank $n$.

Proposition 5. Let $(T, \diamond)$ be a ternary semigroup and $f$ be an n-ary operation on $T$ satisfying the conditions

(i) $f\left(x^{n}\right)=x$ for all $x \in T$,

(ii) $\diamond(f(\bar{x}), x, y)=f\left(\diamond\left(x_{1}, x, y\right), \diamond\left(x_{2}, x, y\right), \ldots, \diamond\left(x_{n}, x, y\right)\right)$ for all $x, x_{i}, y \in T, i=1, \ldots, n$.

Then $(T, \diamond)$ is a diagonal ternary semigroup of some ternary Menger algebras of rank $n$.

Proof. Assume that $(T, \diamond)$ satisfies all the above conditions. We define a $(2 n+1)$-ary operation $\bullet$ on $T$ by

$$
x[\bar{x} \bar{y}]=\diamond(x, f(\bar{y}), f(\bar{z})) \quad \text { for all } x, y_{i}, z_{i} \in T, i=1, \ldots, n .
$$


Now, we show that the operation $\bullet$ is ternary superassociative. Indeed, for $a_{i}, b_{i}, x, y_{i}, z_{i} \in$ $T, i=1, \ldots, n$ we obtain

$$
\begin{aligned}
& x[\bar{y} \bar{z}][\bar{a} \bar{b}]=\diamond(\diamond(x, f(\bar{y}), f(\bar{z})), f(\bar{a}), f(\bar{b})) \\
& =\diamond(x, \diamond(f(\bar{y}), f(\bar{z}), f(\bar{a})), f(\bar{b})) \\
& =\diamond\left(x, f\left(\diamond\left(y_{1}, f(\bar{z}), f(\bar{a})\right), \ldots, \diamond\left(y_{n}, f(\bar{z}), f(\bar{a})\right)\right), f(\bar{b})\right) \\
& =\diamond\left(x, f\left(y_{1}[\bar{z} \bar{a}], \ldots, y_{n}[\bar{z} \bar{a}]\right), f(\bar{b})\right) \\
& =x\left[y_{1}[\bar{z} \bar{a}] \ldots y_{n}[\bar{z} \bar{a}] \bar{b}\right] \quad \text { and } \\
& x\left[\bar{y} z_{1}[\bar{a} \bar{b}] \ldots z_{1}[\bar{a} \bar{b}]\right] \\
& =\diamond\left(x, f(\bar{y}), f\left(z_{1}[\bar{a} \bar{b}], \ldots, z_{n}[\bar{a} \bar{b}]\right)\right) \\
& =\diamond\left(x, f(\bar{y}), f\left(\diamond\left(z_{1}, f(\bar{a}), f(\bar{b})\right), \ldots, \diamond\left(z_{n}, f(\bar{a}), f(\bar{b})\right)\right)\right) \\
& =\diamond(x, f(\bar{y}), \diamond(f(\bar{z}), f(\bar{a}), f(\bar{b}))) \\
& =\diamond(\diamond(x, f(\bar{y}), f(\bar{z})), f(\bar{a}), f(\bar{b})) \\
& =x[\bar{y} \bar{z}][\bar{a} \bar{b}] \text {. }
\end{aligned}
$$

Consequently, $(T, \bullet)$ is a ternary Menger algebra of rank $n$.

Finally, if $(T, *)$ is a diagonal ternary semigroup of this ternary Menger algebra of rank $n$, then for each $x, y, z \in T$ we have

$$
*(x, y, z)=x\left[y^{n} z^{n}\right]=\diamond(x, f(y, \ldots, y), f(z, \ldots, z))=\diamond(x, y, z) .
$$

Hence $(T, *)=(T, \diamond)$, i.e., $(T, \diamond)$ is a diagonal ternary semigroup of $(T, \bullet)$. This proves the proposition.

Corollary 1. Let $(T, \diamond)$ be a ternary semigroup together with a left unit, $(T, \diamond)$ is a diagonal ternary semigroup of some ternary Menger algebras of rank $n$ with a left diagonal unit if and only if there exists an n-ary operation on $T$ which satisfies the conditions (i) and (ii) of Proposition 5.

Proof. Suppose that $(T, \diamond)$ is a diagonal ternary semigroup of a ternary Menger algebra of rank $n$ with a left diagonal unit $e$. We now define an $n$-ary operation $f: T^{n} \longrightarrow T$ by

$$
f(\bar{x})=e\left[e^{n} \bar{x}\right] \quad \text { for all } x_{i} \in T, i=1, \ldots, n .
$$

Indeed, for each $x, x_{i}, y \in T, i=1, \ldots, n$ we obtain $f(x, \ldots, x)=e\left[e^{n} x^{n}\right]=x$ and

$$
\begin{aligned}
\diamond\left(f\left(x_{1}, \ldots, x_{n}\right), x, y\right) & =\diamond\left(e\left[e^{n} \bar{x}\right], x, y\right) \\
& =e\left[e^{n} \bar{x}\right]\left[x^{n} y^{n}\right] \\
& =e\left[e^{n} x_{1}\left[x^{n} y^{n}\right] \ldots x_{n}\left[x^{n} y^{n}\right]\right] \\
& =f\left(x_{1}\left[x^{n} y^{n}\right], \ldots, x_{n}\left[x^{n} y^{n}\right]\right) \\
& =f\left(\diamond\left(x_{1}, x, y\right), \ldots, \diamond\left(x_{n}, x, y\right)\right),
\end{aligned}
$$

which means that $f$ satisfies the conditions (i) and (ii) of Proposition 5.

Conversely, we assume on a ternary semigroup $(T, \diamond)$ with a left unit there exists an $n$-ary operation $f$ satisfying all the conditions of Proposition 5 . Then, this ternary semigroup is also a diagonal ternary semigroup of a ternary Menger algebra $(T, \bullet)$ of rank $n$ under the $(2 n+1)$-ary operation defined by

$$
x[\bar{y} \bar{z}]=\diamond(x, f(\bar{y}), f(\bar{z})) \text { for all } x_{i} \in T, i=1, \ldots, n .
$$

Finally, we suppose that $e$ belongs to this ternary semigroup $T$ be a left unit. Indeed, for $x \in T$ we have

$$
e\left[e^{n} x^{n}\right]=\diamond(e, f(e, \ldots, e), f(x, \ldots, x))=\diamond(e, e, x)=x,
$$


which means that $e$ is a left diagonal unit of a ternary Menger algebra $(T, \bullet)$ of rank $n$.

Next, we give the following example which satisfies Proposition 5 and Corollary 1.

Example 6. Consider the closed interval $I=[0,1]$ together with the ternary operation $\diamond$ defined $b y \diamond(x, y, z)=x$ for all $x, y, z \in I$. It is obvious that $(I, \diamond)$ forms a ternary semigroup with all elements are a left unit. Next, we define an n-ary operation $f$ on I by

$$
f(\bar{x})=\min \{\bar{x}\} \quad \text { for all } x_{i} \in I, i=1, \ldots, n .
$$

Indeed, for each $x, x_{i}, y, z \in I, i=1, \ldots, n$ we get $f\left(x^{n}\right)=\min \left\{x^{n}\right\}=x$ and $\diamond(f(\bar{x}), y, z)=$ $f(\bar{x})=f\left(\diamond\left(x_{1}, y, z\right), \ldots, \diamond\left(x_{n}, y, z\right)\right)$. Hence $f$ satisfies the conditions of Proposition 5. By Corollary 1 , such ternary semigroup is a diagonal ternary semigroup of a ternary Menger algebra $(I, \bullet)$ of rank $n$ with all elements are a left diagonal unit, where the operation $\bullet$ is defined as (5).

Definition 4. Let $\left(S, \bullet_{1}\right)$ and $\left(T, \bullet_{2}\right)$ be two ternary Menger algebras of rank $n$ and let $h: S \longrightarrow$ $T$. Then $h$ is said to be a homomorphism of $S$ into $T$ if

$$
h\left(\bullet_{1}(x, \bar{y}, \bar{z})\right)=\bullet_{2}\left(h(x), h\left(y_{1}\right) \ldots, h\left(y_{n}\right), h\left(z_{1}\right), \ldots, h\left(z_{n}\right)\right)
$$

for all $x, y_{i}, z_{i} \in S, i=1, \ldots, n$.

Furthermore, a homomorphism $h$ of $S$ into $T$ is said to be:

(i) a monomorphism if it is injective;

(ii) an epimorphism if it is surjective;

(iii) an isomorphism if it is bijective, in this case $S$ and $T$ are called isomorphic and written as $T \cong S$. Moreover, if $S$ and $T$ are two ternary Menger algebras with identity elements $e_{S}$ and $e_{T}$ respectively, then $h\left(e_{S}\right)=e_{T}$.

Please note that if $T$ is a ternary semigroup, then we can define two $(2 n+1)$-ary operations on $T$ and we also have $T$ under these operations form two ternary Menger algebras of rank $n$ such that non-isomorphic. Moreover, these ternary Menger algebras may have the same diagonal ternary semigroup although these are non-isomophic.

Example 7. Let $(T, \diamond)$ be a nontrivial commutative ternary semigroup. Then we consider two $(2 n+1)$-ary operations which are defined by

$$
\begin{aligned}
& \bullet_{1}(x, \bar{y}, \bar{z})=\diamond\left(x, y_{1}, z_{1}\right) \text { and } \\
& \bullet_{2}(x, \bar{y}, \bar{z})=\diamond\left(x, y_{2}, z_{2}\right) \text { for all } x, y_{i}, z_{i} \in T, i=1, \ldots, n
\end{aligned}
$$

It is easy to check that $\left(T, \bullet_{1}\right)$ and $\left(T, \bullet_{2}\right)$ are non-isomorphic ternary Menger algebras of rank $n$ together with the same diagonal ternary semigroup $(T, *)$ such that the ternary operation $*$ on $T$ is defined as (4).

\section{Congruence on Ternary Menger Algebra}

In this section, we introduce the concept of congruences and establish quotient structures via congruence on a ternary Menger algebra of rank $n$. Furthermore, we investigate homomorphism and algebraic properties of the quotient structure with respect to congruences are provided.

Definition 5. A binary relation $\rho$ defined on a ternary Menger algebra $(T, \bullet)$ of rank $n$ is called

(i) stable if for all $a, a_{i}, b, b_{i}, c_{i}, d_{i} \in T, i=1, \ldots, n$

$$
(a, b),\left(a_{1}, b_{1}\right), \ldots,\left(a_{n}, b_{n}\right),\left(c_{1}, d_{1}\right), \ldots,\left(c_{n}, d_{n}\right) \in \rho \Longrightarrow(a[\bar{a} \bar{c}], b[\bar{b} \bar{d}]) \in \rho,
$$

(ii) l-regular if for all $a, b, c_{i}, d_{i} \in T, i=1, \ldots, n$

$$
(a, b) \in \rho \Longrightarrow(a[\bar{c} \bar{d}], b[\bar{c} \bar{d}]) \in \rho,
$$


(iii) v-regular if for all $a, b, c_{i}, d \in T, i=1, \ldots, n$

$$
\left(a_{1}, b_{1}\right), \ldots,\left(a_{n}, b_{n}\right) \in \rho \Longrightarrow(d[\bar{c} \bar{a}], d[\bar{c} \bar{b}]) \in \rho,
$$

(iv) $c$-regular if for all $a, b, c_{i}, d \in T, i=1, \ldots, n$

$$
\left(a_{1}, b_{1}\right), \ldots,\left(a_{n}, b_{n}\right) \in \rho \Longrightarrow(d[\bar{a} \bar{c}], d[\bar{b} \bar{c}]) \in \rho,
$$

(v) s-regular if for all $a, b, x, y \in T$

$$
(a, b) \in \rho \Longrightarrow\left(x\left[y^{n} a^{n}\right], x\left[y^{n} b^{n}\right]\right) \in \rho,
$$

(vi) i-regular if for all $a, b, c \in T, \overline{\bar{d}}=\left(d_{1}, d_{2}, \ldots, d_{2 n}\right) \in T^{2 n}, i=1, \ldots, 2 n$

$$
(a, b) \in \rho \Longrightarrow\left(c\left[\left.\overline{\bar{d}}\right|_{i} a\right], c\left[\left.\overline{\bar{d}}\right|_{i} b\right]\right) \in \rho
$$

According to Definition 5, lead us to consider on a ternary semigroup. Then we obtain the conditions (ii) $l$-regular and (vi) $i$-regular are the same thing. Furthermore, we also have the conditions (iii) $v$-regular and (v) s-regular are the same thing.

Definition 6. Let $(T, \bullet)$ be a ternary Menger algebra of rank $n$. An equivalence relation $\rho$ on $T$ is called congruence if it is stable, i.e., for all $a, a_{i}, b, b_{i}, c_{i}, d_{i} \in T, i=1, \ldots, n$

$$
(a, b),\left(a_{1}, b_{1}\right), \ldots,\left(a_{n}, b_{n}\right),\left(c_{1}, d_{1}\right), \ldots,\left(c_{n}, d_{n}\right) \in \rho \Longrightarrow(a[\bar{a} \bar{c}], b[\bar{b} \bar{d}]) \in \rho .
$$

Definition 7. Let $\left(S, \bullet_{1}\right)$ and $\left(T, \bullet_{2}\right)$ be two ternary Menger algebras of rank $n$ and let $h: S \longrightarrow T$ be a homomorphism. Define a relation $\mathrm{ker} h$ on $S$ by

$$
\operatorname{ker} h=\{(a, b) \in S \times S: h(a)=h(b)\},
$$

which is called the kernel of $h$.

From Definition 7, it is easily to seen that ker $h$ is a congruence on $S$.

Let $\rho$ be an equivalence relation on a set $X$ and $X / \rho=\{x \rho: x \in X\}$, where $x \rho=\{y \in$ $X:(x, y) \in \rho\}$. Then we call the set $X / \rho$, a quotient set of $X$ by $\rho$. Moreover, if $(T, \bullet)$ is a ternary Menger algebra of $\operatorname{rank} n$ and $\rho$ is a congruence on $T$, then $T / \rho$ is a ternary Menger algebra of rank $n$ under a $(2 n+1)$-ary operation $\star$ which is defined by

$$
\star\left(x \rho, y_{1} \rho, \ldots, y_{n} \rho, z_{1} \rho, \ldots, z_{n} \rho\right)=x[\bar{y} \bar{z}] \rho \quad \text { for all } x, y_{i}, z_{i} \in T, i=1, \ldots, n .
$$

Then we define a function $\rho^{\natural}: T \longrightarrow T / \rho$ by

$$
\rho^{\natural}(x)=x \rho \quad \text { for all } x \in T \text {. }
$$

It is easily seen that $\rho^{\natural}$ is an epimorphism. We call a function $\rho^{\natural}: T \longrightarrow T / \rho$, the natural homomorphism.

Theorem 1. Let $\left(A, \bullet_{1}\right),\left(B, \bullet_{2}\right)$ and $\left(C, \bullet_{3}\right)$ be three ternary Menger algebras of rank $n$. Let $h: A \longrightarrow B$ and $g: A \longrightarrow C$ be two homomorphisms such that $g$ is surjective. Then

(1) There exists a function $f: C \longrightarrow B$ such that $f \circ g=h$ if and only if $\operatorname{ker} g \subseteq \operatorname{ker} h$.

(2) The function $f$ in (1) is unique.

(3) The function $f$ in (1) is injective if and only if $\operatorname{ker} g=\operatorname{ker} h$.

(4) The function $f$ in (1) is surjective if and only if $h$ is surjective.

Proof. The proof is straightforward.

Theorem 2. Let $\left(S, \bullet_{1}\right)$ and $\left(T, \bullet_{2}\right)$ be two ternary Menger algebras of rank $n$ and $\phi: S \longrightarrow T$ be a surjective homomorphism. Then there exists an isomorphism $h$ from $S / \operatorname{ker} \phi$ onto $T$ which is unique such that $h \circ(\operatorname{ker} \phi)^{\natural}=\phi$. 
Proof. The proof follows from Theorem 1.

Theorem 3. Let $\left(S, \bullet_{1}\right)$ and $\left(T, \bullet_{2}\right)$ be two ternary Menger algebras of rank $n$ and let $\rho$ be a congruence on $S$. Let $\phi: S \longrightarrow T$ be a homomorphism such that $\rho \subseteq \operatorname{ker} \phi$. Then there exists a unique homomorphism $f: S / \rho \longrightarrow T$ with $\operatorname{Im} f=\operatorname{Im} \phi$ and the diagram

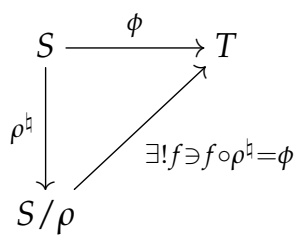

is commutative, i.e., $f \circ \rho^{\natural}=\phi$ where $\rho^{\natural}$ is a natural homomorphism.

Proof. The proof is straightforward.

Theorem 4. Let $(T, \bullet)$ be a ternary Menger algebra of rank $n$ and let $\rho$ and $\sigma$ be congruence on $T$ such that $\rho \subseteq \sigma$. Then there exists a unique surjective homomorphism $g: T / \rho \longrightarrow T / \sigma$ such that $g \circ \rho^{\natural}=\sigma^{\natural}$, i.e., the following diagram

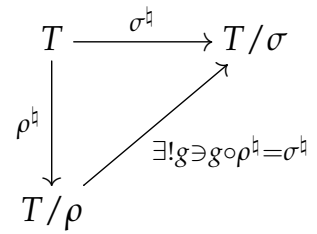

is commutative, i.e., $g \circ \rho^{\natural}=\sigma^{\natural}$ where $\rho^{\natural}$ and $\sigma^{\natural}$ are natural homomorphisms.

Proof. The proof is similar to Theorem 3.

As an immediately consequence of Theorem 4, we have the following results:

Corollary 2. Let $(T, \bullet)$ be a ternary Menger algebra of rank $n$ and let $\rho_{1}, \rho_{2}, \ldots, \rho_{m}(m \geq 2)$ be congruences on $T$ such that $\rho_{1} \subseteq \rho_{2} \subseteq \cdots \subseteq \rho_{m}$. Then there exists a surjective homomorphism $f: T / \rho_{1} \longrightarrow T / \rho_{m}$.

Theorem 5. Let $\rho$ and $\sigma$ be two congruence on a ternary Menger algebra $(T, \bullet)$ of rank $n$ such that $\rho \subseteq \sigma$. Then $\sigma / \rho=\{(a \rho, b \rho) \in T / \rho \times T / \rho:(a, b) \in \sigma\}$ is a congruence on $T / \rho$ and $(T / \rho) /(\sigma / \rho) \cong T / \sigma$.

Proof. The proof follows from Theorem 4 .

Corollary 3. Let $T$ be a ternary Menger algebra of rank $n$ and let $\rho_{1}, \rho_{2}, \ldots, \rho_{m}, \rho_{m+1}$ be congruence on $T$ such that $\rho_{1} \subseteq \rho_{2} \subseteq \cdots \subseteq \rho_{m} \subseteq \rho_{m+1}$. Then for each $i=1, \ldots, m$,

$$
\rho_{i+1} / \rho_{i}=\left\{\left(a \rho_{i}, b \rho_{i}\right) \in T / \rho_{i} \times T / \rho_{i}:(a, b) \in \rho_{i+1}\right\}
$$

is a congruences on $T / \rho_{i}$ and $\left(T / \rho_{i}\right) /\left(\rho_{i+1} / \rho_{i}\right) \cong T / \rho_{i+1}$.

Furthermore, for each $i=1, \ldots, m-1$, the mapping

$$
\phi_{i}:\left(T / \rho_{i}\right) /\left(\rho_{i+1} / \rho_{i}\right) \longrightarrow\left(T / \rho_{i+1}\right) /\left(\rho_{i+2} / \rho_{i+1}\right)
$$

is a surjective homomorphism.

Please note that Corollary 3 is a generalization of the Theorem 5, i.e., if we set a natural number $m=1$, then we get Corollary 3 and Theorem 5 are the same. Again, if we set a 
natural number $n=m=1$, then Corollary 3 is a generalization of the second isomorphism theorem for ternary semigroups (see Theorem 3.7, [34]).

\section{Reduction of Ternary Menger Algebra into Menger Algebra}

In this section, we are about to show that a ternary Menger algebra of rank $n$ together with some conditions can be reduced to a Menger algebra of rank $n$. In addition, it can be reduced even into a band with some specific conditions.

Lemma 1. Let $(T, \bullet)$ be a ternary Menger algebra of rank $n$ which admits an idempotent element a satisfying the following properties; for $i=1, \ldots, n$

(a) (i) $a\left[a^{n} x^{n}\right]=a\left[x^{n} a^{n}\right]=x\left[a^{n} a^{n}\right]=x$ for all $x \in T$

(ii) $a\left[x^{n} \bar{y}\right] \in T$ for all $x, y_{i} \in T$ such that $x \neq y_{i}$ and $x, y_{i} \neq a$

$$
a\left[x^{n} x^{n}\right]=x\left[a^{n} x^{n}\right]=x\left[x^{n} a^{n}\right]=x \text { for all } x \in T
$$

Then $(\beta)$ implies $(\alpha)$.

Proof. $(\beta)$ implies $(\alpha) \quad(i)$ : Indeed, for $x \in T$ we have

$$
\begin{aligned}
& a\left[a^{n} x^{n}\right]=a\left[a^{n} a\left[x^{n} x^{n}\right] \ldots a\left[x^{n} x^{n}\right]\right]=a\left[a^{n} a^{n}\right]\left[x^{n} x^{n}\right]=a\left[x^{n} x^{n}\right]=x, \\
& a\left[x^{n} a^{n}\right]=a\left[a\left[x^{n} x^{n}\right] \ldots a\left[x^{n} x^{n}\right] a^{n}\right]=a\left[a^{n} x^{n}\right]\left[x^{n} a^{n}\right]=x\left[x^{n} a^{n}\right]=x, \\
& x\left[a^{n} a^{n}\right]=x\left[x^{n} a^{n}\right]\left[a^{n} a^{n}\right]=x\left[x^{n} a\left[a^{n} a^{n}\right] \ldots a\left[a^{n} a^{n}\right]\right]=x\left[x^{n} a^{n}\right]=x .
\end{aligned}
$$

( $\beta$ ) implies $(\alpha)$ (ii): Indeed, for $x, y_{i} \in T, i=1, \ldots, n$ we have

$$
a\left[x \ldots x y_{1} \ldots y_{n}\right]=a\left[a\left[x^{n} x^{n}\right] \ldots a\left[x^{n} x^{n}\right] b_{1} \ldots b_{n}\right]=a\left[a^{n} x^{n}\right]\left[x \ldots x y_{1} \ldots y_{n}\right]=x\left[x \ldots x y_{1} \ldots y_{n}\right] \in T .
$$

Please note that on the other hand, $(\alpha)$ implies $a\left[x^{n} x^{n}\right]=x\left[a^{n} x^{n}\right]=x\left[x^{n} a^{n}\right]$ only. Indeed, for $x \in T$ we obtain

$$
a\left[x^{n} x^{n}\right]=a\left[a\left[x^{n} a^{n}\right] \ldots a\left[x^{n} a^{n}\right] a\left[x^{n} a^{n}\right] \ldots a\left[x^{n} a^{n}\right]\right]=a\left[a^{n} x^{n}\right]\left[a^{n} x^{n}\right]=x\left[a^{n} x^{n}\right] \text { and } x\left[a^{n} x^{n}\right]=
$$
$x\left[a \ldots a a\left[x^{n} a^{n}\right] \ldots a\left[x^{n} a^{n}\right]\right]=x\left[a^{n} a^{n}\right]\left[x^{n} a^{n}\right]=x\left[x^{n} a^{n}\right]$.

Consequently, $a\left[x^{n} x^{n}\right]=x\left[a^{n} x^{n}\right]=x\left[x^{n} a^{n}\right]$. The counterexample is already shown in Example 4.16 in [46] where we consider a ternary semigroup as a ternary Menger algebra of rank 1.

Lemma 2. Let $(T, \bullet)$ be a ternary Menger algebra of rank $n$ which admits an idempotent element a satisfying the properties $(\alpha)$ and $(\lambda)$ which is given below

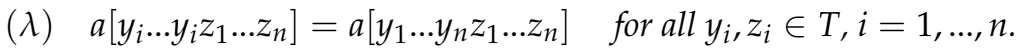

Then $T$ is a Menger algebra of rank $n$ under an $(n+1)$-ary operation o which is defined by

$$
o(x, \bar{y})=a\left[x^{n} \bar{y}\right] \quad \text { for all } x, y_{i} \in T, i=1, . ., n
$$

and $x[\bar{y} \bar{z}]=o(o(x, \bar{y}), \bar{z})$ for all $x, y_{i}, z_{i} \in T, i=1, \ldots, n$.

Proof. Assume that the hypothesis of the theorem is true. First, we define an $(n+1)$-ary operation $o$ on $T$ by

$$
o(x, \bar{y})=a\left[x^{n} \bar{y}\right] \quad \text { for all } x, y_{i} \in T, i=1, \ldots, n .
$$

By the property (ii) of $(\alpha)$ holds, we have $o$ is an $(n+1)$-ary operation on $T$ and $T$ is closed under this operation. Here we use the fact that $T$ is a ternary Menger algebra of rank $n$ and the properties (ii) of $(\alpha)$ and $(\lambda)$ hold. Indeed, for $x, y_{i}, z_{i} \in T, i=1, . ., n$ we obtain 


$$
\begin{aligned}
o(o(x, \bar{y}), \bar{z}) & =o\left(a\left[x \ldots x y_{1} \ldots y_{n}\right], z_{1}, \ldots, z_{n}\right) \\
& =a\left[a\left[x \ldots x y_{1} \ldots y_{n}\right] \ldots a\left[x \ldots x y_{1} \ldots y_{n}\right] z_{1} \ldots z_{n}\right] \\
& =a\left[a^{n} x^{n}\right]\left[y_{1} \ldots y_{n} z_{1} \ldots z_{n}\right] \\
& =x[\bar{y} \bar{z}] \quad \text { and } \\
o\left(x, o\left(y_{1}, \bar{z}\right), \ldots, o\left(y_{n}, \bar{z}\right)\right) & =o\left(x, a\left[y_{1} \ldots y_{1} z_{1} \ldots z_{n}\right], \ldots, a\left[y_{n} \ldots y_{n} z_{1} \ldots z_{n}\right]\right) \\
& =a\left[x \ldots x a\left[y_{1} \ldots y_{1} z_{1} \ldots z_{n}\right] \ldots a\left[y_{n} \ldots y_{n} z_{1} \ldots z_{n}\right]\right] \\
& =a\left[x \ldots x a\left[y_{1} \ldots y_{n} z_{1} \ldots z_{n}\right] \ldots a\left[y_{1} \ldots y_{n} z_{1} \ldots z_{n}\right]\right] \\
& =a\left[x^{n} a^{n}\right]\left[y_{1} \ldots y_{n} z_{1} \ldots z_{n}\right] \\
& =x[\bar{y} \bar{z}] .
\end{aligned}
$$

It follows that $o$ is superassociative and hence $(T, o)$ is a Menger algebra of rank $n$.

From Lemma 2, let us consider on reduction of ternary semigroup into semigroup. The condition $(\lambda)$ is not necessary to suppose it because it is true on ternary semigroups.

Lemma 3. Let $(T, \bullet)$ be a ternary Menger algebra of rank $n$ which admits an idempotent element a satisfying the property $(\beta)$, then there exists an $(n+1)$-ary operation o on $T$ such that $(T, o)$ is band (a Menger algebra of rank $n$ with $o(x, x, \ldots, x)=x$ for all $x \in T$ ) and $x\left[y_{1} \ldots y_{n} z_{1} \ldots z_{n}\right]=$ $o\left(o\left(x, y_{1}, \ldots, y_{n}\right), z_{1}, \ldots, z_{n}\right)$ for all $x, y_{i}, z_{i} \in T, i=1, \ldots, n$.

Proof. From Lemma 1, we get $(\beta)$ implies $(\alpha)$. Then the $(n+1)$-ary operation $o: T^{n+1} \longrightarrow$ $T$ defined by

$$
o\left(x, y_{1}, \ldots, y_{n}\right)=a\left[x \ldots x y_{1} \ldots y_{n}\right] \quad \text { for all } x, y_{i} \in T, i=1, . ., n
$$

is superassociative. Hence $(T, o)$ forms a Menger algebra of rank $n$.

Again, by Lemma 1, we obtain $x\left[y_{1} \ldots y_{n} z_{1} \ldots z_{n}\right]=o\left(o\left(x, y_{1}, \ldots, y_{n}\right), z_{1}, \ldots, z_{n}\right)$ for all $x, y_{i}, z_{i} \in T, i=1, \ldots, n$. Moreover, for each $x \in T$ we have

$$
o(x, x, \ldots, x)=a\left[x^{n} x^{n}\right]=x,
$$

which implies that $x$ is an idempotent element and hence $(T, o)$ is band.

\section{Conclusions and Future Works}

We introduced the notion of ternary Menger algebra of rank $n$ which can be regard as a canonical generalization of arbitrary ternary semigroups. Based on this knowledge, we established the so-called the diagonal ternary semigroup and investigated its interesting algebraic properties. Next, we continued by introduction of the notion of congruences on ternary Menger algebras. Moreover, the homomorphism theorem for ternary Menger algebras is investigated. Furthermore, by using some additional conditions, we showed the reduction of ternary Menger algebra into Menger algebra (a generalization of arbitrary semigroups).

In future work, we will discuss several ideal theories for ternary Menger algebras and some applications to fuzzy theory, and we will extend some well-known results related to arbitrary ternary semigroups to extension of ternary Menger algebras. Moreover, we will establish the representation of ternary Menger algebras for further development of its algebraic structures.

Author Contributions: All two authors contributed equally to all aspects of this work. All authors have read and agreed to the published version of the manuscript.

Funding: This research received no external funding.

Acknowledgments: The authors are highly grateful to the referees for their valuable comments and suggestions for improving the article. This research was supported by Chiang Mai University, Chiang Mai 50200, Thailand.

Conflicts of Interest: The authors declare no conflict of interest. 


\section{References}

1. Menger, K. General algebra of analysis. Rep. Math. Colloq. Notre Dame Univ. 1946, 7, 46-60.

2. Schein, B.M. Theory of semigroups as theory of superpositions of multiplace functions. In Proceedings of the Inter-University Symposium in General Algebra; Tartu State University: Tartu, Estonia, 1966; pp. 169-192.

3. Dudek, W.A.; Trokhimenko, V.S. Congruences on Menger algebras. Comm. Algebra 2014, 42, 3407-3426. [CrossRef]

4. Dudek, W.A.; Trokhimenko, V.S. On $\sigma$-Commutativity in Menger algebras of $n$-plac functions. Comm. Algebra 2017, 45, 4557-4568. [CrossRef]

5. Trokhiemko, V.S. v-regular Menger algebras. Algebra Univ. 1997, 38, 150-164. [CrossRef]

6. Denecke, K. Regular Elements and Green's Relations in Menger Algebras of Terms. Discuss. Math.-Gen. Algebra Appl. 2006, 26, 85-109. [CrossRef]

7. Clifford, A.H. Extensions of semigroups. Trans. Am. Math. Soc. 1950, 68, 165-173. [CrossRef]

8. Schein, B.M. On Translations in semiqroups and groups (Russian). Volzski Matem. Sbornik 1964, 2, $163-169$.

9. Kumduang, T.; Leeratanavalee, S. Left Translations and Isomorphism Theorems for Menger algebras of Rank $n$. Kyungpook Math J. 2020, accepted.

10. Kumduang, T.; Leeratanavalee, S. Menger hyperalgebras and their representations. Comm. Algebra 2020, 1-21. [CrossRef]

11. Dudek, W.A.; Trokhimenko, V.S. Algebras of Multiplace Functions; Walter de Gruyter GmbH \& Co. KG.: Berlin, Germany; Boston, MA, USA, 2012; pp. 21-84.

12. Lehmer, D.H. A Ternary Analogue of Abelian groups. Am. J. Math. 1932, 54, 329-338. [CrossRef]

13. Kanser, E. An extension of the group concept. Bull. Am. Math. Soc. 1904, 10, 290-291.

14. Ashrafi, N.; Yazdanmehr, Z. *-Relations on Ternary Semigroups. Int. J. Geom. Methods Mod. Phys. 2018, 15, 1850094. [CrossRef]

15. Kerner, R. Ternary Algebraic Structures and Their Applications in Physics; Pierre and Marie Curie University: Paris, France, 2000.

16. Nambu, Y. Generalized Hamiltonian dynamics. J. Phys. Rev. 1973, 7, 2405. [CrossRef]

17. Amyari, M.; Moslehian, M.S. Approximate Homomorphisms of Ternary Semigroups. Lett. Math. Phys. 2006, 77, 1-9. [CrossRef]

18. Gordji, M.E.; Jabbari, A.; Ebadian, A.; Ostadbashi, S. Automatic Continuity of 3-Homomorphisms on Ternary Banch Algebras. Int. J. Geom. Methods Mod. Phys. 2013, 10, 13200012.

19. Rassias, T.M.; Brzdek, J. Functional Equations in Mathematical Analysis; Springer: New York, NY, USA, 2012 ; pp. $372-416$.

20. Ataguema, H.; Makhlouf, A. Notes on cohomologies of ternary algebras of associative type. J. Gen. Lie Theory Appl. 2009, 3, 157-174. [CrossRef]

21. Bazunova, N.; Borowiec, A.; Kerner, R. Universal differential calculus on ternary algebras. Lett. Math. Phys. 2004, 67, 195-206. [CrossRef]

22. Chronowski, A. Ternary linear algebras and topological ternary structures. Tr. Inst. Prikl. Mat. Mekh. 2005, 11, 62-70.

23. Hyers, D.H.; Rassias, T.M. Approximate homomorphisms. Aequationes Math. 1992, 44, 125-153. [CrossRef]

24. Park, C. Generalized Hyers-Ulam stability of $C^{*}$-ternary algebra homomorphisms. Dyn. Contin. Discrete Impuls. Syst. Ser. A Math. Anal. 2009, 16, 67-79.

25. Park, K.H.; Jung, Y.S. Perturbations of Higher ternary derivations in Banach ternary algebras. Commun. Korean Math. Soc. 2008, 23, 387-399. [CrossRef]

26. Savadkouhi, M.B.; Gordji, M.E.; Rassias, J.M.; Ghobadipour, N. Approximate ternary Jordan derivations on Banach ternary algebras. J. Math. Phys. 2009, 50, 042303. [CrossRef]

27. Chronowski, A. On the conditional Cauchy equation on 3-adic groups. Zb. Rad. Prirod. Mat. Fak. Ser. Mat. 1993, $23,269-282$.

28. Chronowski, A. On the Pexider equation and the Cauchy equation on 3-adic groups. Wyz. Szkoła Ped. Kraków Rocznik Nauk. Dydakt 1987, 115, 173-176.

29. Hosszú, M. On the explicit forḿ of n-group operations. Publ. Math. Debrecen 1963, 10, 88-92.

30. Park, C.G.; Rassias, T.M. Homomorphisms in $C^{*}$-ternary algebras and JB*-triples. J. Math. Anal. Appl. 2008, 337, 13-20. [CrossRef]

31. Park, C.; Rassias, T.M. Homomorphisms and derivations in proper JCQ*-triples. J. Math. Anal. Appl. 2008, 337, 1404-1414. [CrossRef]

32. Rassias, J.M.; Kim, H.M. Approximate homomorphisms and derivations between $C^{*}$-ternary algebras. J. Math. Phys. 2008, 49, 1-10. [CrossRef]

33. Moslehian, M.S.; Rassias, T.M. Generalized Hyers-Ulam stability of mappings on normed Lie triple systems. Math. Inequal. Appl. 2008, 11, 371-380. [CrossRef]

34. Kar, S.; Maity, B.K. Congruences on Ternary Semigroups. Chungcheong Math. Soc. 2007, 20, 191-201.

35. Los, J. On the Extending of Models I. Fundam. Math. 1955, 42, 38-54. [CrossRef]

36. Monk, D.; Sioson, F.M. $m$-Semigroups, Semigroups and Function Representations. Fund. Math. 1966, 59, 233-246. [CrossRef]

37. Sioson, F.M. Ideal theory in Ternary Semigroups. Math. Jpn. 1965, 10, 63-84.

38. Sheeja, G.; Sri Bala, S. Orthodox Ternary Semigroups. Quasigroups Relat. Syst. 2011, 19, 339-348.

39. Vagner, V. The Theory of Generalized heaps and Generalized Groups. Mat. Sb. 1953, 32, 545-632.

40. Santiago, M.L. Some Contributions to the Study of Ternary Semigroups and Semiheaps. Ph.D. Thesis, University of Madras, Chennai, India, 1983.

41. Post, E.L. Polyadic groups. Trans. Am. Math. Soc. 1940, 48, 208-350. [CrossRef]

42. Głazek, K.; Gleichgewicht, B. On some method of construction of the covering group. Acta Univ. Wratislav. 1973, 188, 125-135. 
43. Santiago, M.L.; Sri Bala, S. Ternary Semigroups. Semigroup Forum 2010, 81, 280-388. [CrossRef]

44. Chronowski, A. Congruences on Ternary Semigroups. Ukr. Math. J. 2004, 56, 662-681. [CrossRef]

45. Sheeja, G.; Sri Bala, S. Congruences on Ternary semigroups. Quasigroups Relat. Syst. 2012, 20, 113-124.

46. Dixit, V.N.; Dewan, S. Congruence and Green's Equivalence Relation on Ternary Semigroup. Commun. Fac. Sci. Univ. Ank. Ser. A1-Math. Stat. 1997, 46, 103-117. 